\title{
GPS-based orbital filter to reach the moon
}

\section{Francesco Basile*, Vincenzo Capuano, Cyril Botteron and Pierre-André Farine}

École Polytechnique Fédérale de Lausanne (EPFL),

EPFL STI IMT-NE ESPLAB,

Rue de la Maladière 71B,

CH-2000 Neuchâtel, Switzerland

Email: basile.fran1989@gmail.com

Email: vincenzo.capuano@epfl.ch

Email: cyril.botteron@epfl.ch

Email: pierre-andre.farine@epfl.ch

*Corresponding author

\begin{abstract}
Nowadays, global navigation satellite systems (GNSSs) are used for many new applications that go further than the original goal of providing position, velocity and timing for land, maritime and air applications. In particular, GNSS receivers have been adopted as main navigation system for several low Earth orbits (LEOs) missions, increasing the autonomy of the hosting spacecraft, reducing the networking operation costs. Accordingly, they result in an attractive solution even for higher Earth orbits. However, although many studies have shown that GNSS observations can also be obtained at altitudes above the GNSS constellations by using high sensitivity receivers, the use of these signals is still challenging because of their very weak power and the poor relative geometry between the receiver and the transmitters. In this paper, we describe the implementation of an orbital filter specifically designed for moon missions, which aims to improve the navigation performance achievable when using GNSS observations
\end{abstract}

Keywords: global navigation satellite systems; GNSSs; highly elliptical orbit; HEO; moon transfer orbit; MTO; global positioning system; GPS; orbital filter; Kalman filter.

Reference to this paper should be made as follows: Basile, F., Capuano, V., Botteron, C. and Farine, P-A. (2015) 'GPS-based orbital filter to reach the moon', Int. J. Space Science and Engineering, Vol. 3, No. 3, pp.199-218.

Biographical notes: Francesco Basile is a Space and Astronautical Engineer. $\mathrm{He}$ received his Master degree with full marks at the University of Rome 'La Sapienza' and his Bachelor degree in Aerospace Engineering at the University of Naples 'Federico II'. During his Master thesis, he worked at the École Polytechnique Fédérale de Lausanne (EPFL) as an intern, on an adaptive orbital filter for GNSS receivers in challenging space environment. His research activity focuses on navigation algorithms for space applications.

Vincenzo Capuano is a PhD candidate in Microsystems and Microelectronics at the École Polytechnique Fédérale de Lausanne (EPFL) and doctoral assistant in the GNSS group at EPFL STI IMT ESPLAB, in Neuchâtel, Switzerland. His current research activity focuses on the GNSS use for space navigation. $\mathrm{He}$ received his Master of Engineering in Astronautic Engineering with full marks from the University of Rome 'La Sapienza' and his Bachelor of Engineering in 
Aerospace Engineering with full marks cum laude from the University of Naples 'Federico II'.

Cyril Botteron is leading, managing, and coaching the research and project activities of the Global Navigation Satellite System and Ultra-Wideband and mm-wave groups at École Polytechnique Fédérale de Lausanne (EPFL). He is the author or co-author of five patents and over 80 publications in major journals and conferences in the fields of wireless positioning systems, GNSS-based navigation and sensing, ultra-low-power radio frequency communications and integrated circuits design, and baseband analogue and digital signal processing.

Pierre-André Farine is Professor in Electronics and Signal Processing at EPFL, and is Head of the Electronics and Signal Processing Laboratory. He received his MSc and $\mathrm{PhD}$ in Micro Technology from the University of Neuchâtel, Switzerland, in 1978 and 1984, respectively. He is active in the study and implementation of low-power solutions for applications covering wireless telecommunications, ultra-wideband, global navigation satellite systems, and video and audio processing. He is the author or co-author of more than 100 publications in conference and technical journals and 50 patent families (more than 270 patents).

\section{Introduction}

Improvements in signal processing techniques and deep sub-microns CMOS integrated circuits design have resulted in tremendous progresses in global navigation satellite system (GNSS) receiver technology and are now allowing its use for applications in very challenging environments, such as the space environment. Indeed, although not specifically designed for spacecrafts, GNSS is today widely used for attitude determination, time synchronisation, orbit determination, and absolute and relative position determination in low Earth orbits (LEOs), as its use can significantly reduce the burden and costs of the networking operations and increase the autonomy of the hosting spacecraft (Bauer et al., 2006).

Promising experimental results presented in Powell et al. (1999) and Balbach et al. (1998) have demonstrated that by modifying the traditional signal processing techniques, GNSS can also be used in higher orbits such as medium Earth orbit (MEO) and high Earth orbit (HEO). More recent research studies have shown the interest of the scientific space community to investigate the potential use of GNSS as navigation system for lunar missions (Manzano-Jurado et al., 2014; Silva et al., 2013; Capuano et al., 2014a, 2014b; Palmerini et al., 2009). Being its service originally conceived for Earth applications, the GNSS transmitters point towards the Earth, making their transmitted signals very weak above the GNSS constellation. However, some of these first studies (see e.g., Manzano-Jurado et al., 2014; Capuano et al., 2014a; Silva et al., 2013) have revealed that although weak, GNSS signals from the side lobes of the GNSS transmitters antennas or from the spillover of the main lobe can still be acquired and tracked successfully. Experimental demonstrations have been presented in Balbach et al. (1998) for HEO, while theoretical studies have been described in Capuano et al. (2014a) for higher orbits up to moon altitude. 
At the same time, such studies have also highlighted how coarse a GNSS standalone navigation solution at moon altitude can be. In particular, the higher the receiver is flying above the GNSS constellation, the weaker the GNSS received signals are (thus affecting the number of visible satellites and the pseudorange accuracy from the visible ones) and the larger the geometric dilution of precision (GDOP) is (resulting in lower positioning accuracy).

However, if GNSS observations are filtered through an orbital dynamics mathematical model that is able to predict the observations themselves, the achievable navigation accuracy can be much higher. Such kind of data fusion is commonly known as 'orbital filter'. Several research papers describe the use of orbital filter for LEO, such as Zhou (2004), Chiaradia et al. (2000), Choi et al. (2010) and Habib (2013).

In this paper, we propose to extend the use of orbital filtering for a moon mission and present the navigation performance achieved by using a global positioning system (GPS)-based orbital filter specifically designed for a moon transfer orbit (MTO). The analysis is carried out for two configurations of the filter: in the first one ('position-based'), the measurement input of the filter are the least squares GPS positions, and velocity, while in the second one ('range-based'), the measurement inputs are directly the pseudorange and pseudorange rates. In order to highlight the benefit of its use, the achieved orbital filter performance is compared to the ones that would be obtained in the same scenario by using a simple least square estimator of the GPS measurements.

\section{Reference lunar mission}

Table 1 reports the initial position and velocity of the receiver trajectory of the considered MTO that is accurately propagated by the PosApp software of our Spirent (2012) GSS8000 simulator. The same table also reports some characteristics assumed for the hosting spacecraft. The resulting trajectory takes into account gravitational effects from the Earth up to the 21st degree and 21st order, sun and moon gravity, solar radiation pressure (SRP) and atmospheric drag, and is represented by the blue portion of the MTO shown in Figure 1.

Table 1 Initial conditions for the MTO and spacecraft characteristics

\begin{tabular}{lc}
\hline Parameters & Values \\
\hline ECI Initial position $(\mathrm{km})$ & {$[2395.52-5298.28-3022.82]$} \\
ECI Initial velocity $(\mathrm{km} / \mathrm{s})$ & {$[10.193 .581 .72]$} \\
Departure date & 2nd July 2005 00:34:18 \\
Mass of the spacecraft $(\mathrm{kg})$ & 1,000 \\
Reference surface $\left(\mathrm{m}^{2}\right)$ & 20 \\
\hline
\end{tabular}

Note: The position and velocity values are expressed in Earth centred inertial (ECI) frame. 
Figure 1 Considered lunar mission: the MTO is the curve in light blue (image from STK) (see online version for colours)

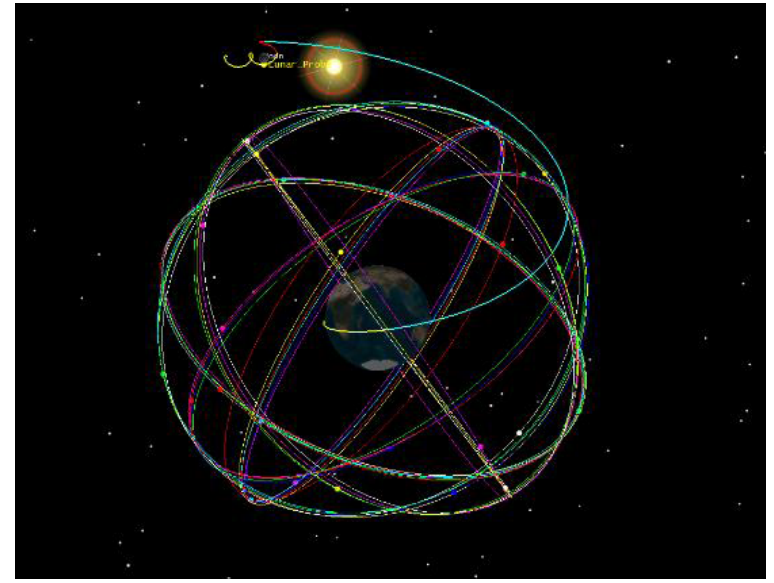

\section{Signal assumptions and measurements model}

Realistic GPS L1 C/A power signals levels at the receiver position are also modelled by the PosApp software of Spirent, according to the GPS interface control document (Global Positioning System Directorate, 2013). In particular, PosApp has been set to simulate a GPS constellation made of 30 GPS satellites, allocated in six orbital planes. Besides, in order to model accurately the 3D antenna patterns of the GPS transmitters, we used the pattern from Block II-A as defined in Czopek and Shollenberger (1993) and provided by Spirent.

Following our previous study of the GPS signals power levels during a MTO (see Capuano et al., 2014a, 2014b), an antenna gain of $10 \mathrm{dBi}$ is assumed in order to be able to acquire and track signals down to $-169 \mathrm{dBm}$ using a $-159 \mathrm{dBm}$ sensitivity GPS receiver. In order to guarantee $10 \mathrm{dBi}$ antenna gain during the whole trajectory a steerable antenna could be used (Litva, 1996) or more than one receiver antenna could be placed on different faces of the spacecraft, with at least one that points to the GPS satellites at all times as proposed in Palmerini et al. (2009).

Table 2 summarises all the assumptions about the transmitted signals and their processing. Such assumptions respect the parameters settings of the WeakHEO receiver, a space borne receiver developed by the EPFL ESPLAB, for which the orbital filter described here has been designed. A short description of such receiver can be found in Capuano et al. (2014b).

For our simulations, the pseudorange and pseudorange rate observables are modelled according to Kaplan and Hegarty (2006), respectively as

$$
\begin{aligned}
& \rho=\sqrt{\left(x_{\text {sat }}-x_{u}\right)^{2}+\left(y_{\text {sat }}-y_{u}\right)^{2}+\left(z_{\text {sat }}-z_{u}\right)^{2}}+b+\text { noise } \\
& \dot{\rho}=\left(\boldsymbol{v}_{\text {sat }}-\boldsymbol{v}_{\boldsymbol{u}}\right) \cdot \boldsymbol{a}+\dot{b}+\text { noise }
\end{aligned}
$$


where in equation (3.1): $x_{\text {sat }}, y_{\text {sat }}, z_{\text {sat }}$ denote the Earth centred inertial (ECI reference frame) position's components of the GPS satellite that is transmitting the signal, $x_{u}, y_{u}, z_{u}$ are the user's ECI position components, and $b$ is the receiver's clock offset. While in (3.2), $\boldsymbol{v}_{\boldsymbol{s a t}}$ and $\boldsymbol{v}_{\boldsymbol{u}}$ are, respectively, the velocity vector of the transmitting GPS satellite and of the spacecraft, $\dot{b}$ represents the clock's drift, and $\boldsymbol{a}$ is the line-of-sight (LOS) unit vector from the user to the GPS satellite. The true positions and velocities of the GPS satellites and of the receiver, which are considered as reference in the simulations are provided by PosApp software.

Table 2 Signal and processing assumption used for the study

\begin{tabular}{lc}
\hline Parameters & Values \\
\hline Processed signal & GPS-L1 C/A \\
Receiver sensitivity $(\mathrm{dBm})$ & -159 \\
Antenna gain $(\mathrm{dBi})$ & 10 \\
Chipping rate $(\mathrm{Mchip} / \mathrm{s})$ & 1.023 \\
Code loop noise bandwidth (Hz) & 0.5 \\
Early-to-late correlator spacing (chip) & 0.1 \\
Coherent integration time (ms) & 20 \\
Double-sided front end bandwidth (MHz) & 26 \\
Front-end figure (dB) & 2 \\
Effective antenna temperature (K) & 130 \\
\hline
\end{tabular}

Note: The value of $26 \mathrm{MHz}$ for the double-sided front end bandwidth is related to the front-end used for testing the WeakHEO receiver.

What is called 'noise' has been modelled as the square root of the sum of the variance of several noise contributions. The corresponding assumed standard deviations for each contribution of the pseudorange noise errors are listed in Table 3. The values have been taken from Kaplan and Hegarty (2006), except for the ionospheric delay and receiver error which are explained below.

Table 3 GPS C/A code error budget for space users

\begin{tabular}{lc}
\hline Error source & l $\sigma$ error $(m)$ \\
\hline Broadcast clock & 1.1 \\
Broadcast ephemeris & 0.8 \\
Ionospheric delay & 15 \\
Tropospheric delay & 0.2 \\
Receiver noise & $\left(0.1^{2}+\sigma_{t D L L}^{2}\right)^{0.5}$ \\
Multipath & 0.2 \\
\hline
\end{tabular}

According to Kaplan and Hegarty (2006), a typical 1-sigma error due to ionospheric effects, averaged over the globe and over elevation angles, for a user on the Earth surface, is $7 \mathrm{~m}$. Such value corresponds to the residual error after the ionosphere corrections are applied [according to the Klobuchar (1987) model]. However, we assume here a more conservative approximate value of $15 \mathrm{~m}$ to take into account the wider range of elevation angles encountered when the receiver is flying in the space above the GPS constellation. 
Regarding the thermal noise code tracking jitter $\sigma_{t D L L}$, it has been modelled to take into account the strong dependence on the carrier to noise ratio $C / N_{0}$. This ratio can reach very small values when the receiver is far away from the GPS constellation (getting closer to the moon altitude), thus inducing a much higher thermal noise code tracking jitter than on the Earth surface. Considering the binary phase shift keying (BPSK) modulation used for the GPA L1 C/A signal and a non-coherent early minus late power DLL discriminator used, the thermal noise code tracking jitter $\sigma_{t D L L}$ can be written as (Betz and Kolodziejski, 2000):

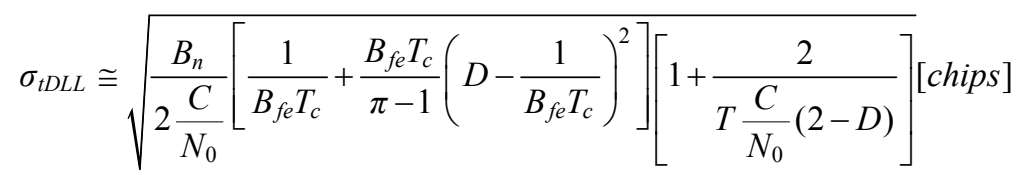

where $B_{n}$ is the code loop noise bandwidth expressed in $\mathrm{Hz} ; D$ is the early-to-late correlator spacing in unit of chips; $T$ is the coherent integration time in seconds; $B_{f e}$ is the double-sided front-end bandwidth in $\mathrm{Hz} ; R_{c}=1 / T_{c}$ is the chipping rate expressed in $\mathrm{Hz} / \mathrm{s}$; and $C / N_{0}$ is the carrier to noise ratio. For the assumed front-end noise figure $(2 \mathrm{~dB})$ and effective antenna temperature $(130 \mathrm{~K})$, the carrier-to-noise ratio is given by (Van Diggelen, 2009):

$$
C / N_{0}=P_{r}+174
$$

where $P_{r}$ is the received power, simulated by Spirent.

Finally, the pseudorange rate is assumed to be computed from the Doppler measurements and its noise will thus be proportional to the Doppler rate noise. The Doppler rate noise has been modelled as Doppler tracking jitter according to Borio et al. (2010), as follows:

$$
\sigma_{f}=\frac{1}{T} \sqrt{\frac{B_{n}}{C / N_{0}}\left(1+\frac{1}{2 T C / N_{0}}\right)}\left[\frac{\mathrm{rad}}{\mathrm{s}}\right]
$$

\section{Stand-alone GPS accuracy}

As already described in Section 3, we have used the Spirent software PosApp to simulate the real positions and velocities of the receiver and of the GPS satellites as well as the signals power level at the receiver position. Such quantities have been used to model the GPS measurements obtained by a $-159 \mathrm{dBm}$ sensitivity GPS receiver during the whole trajectory. Figure 2 illustrates the standalone GPS 3D position accuracy obtained by processing the GPS pseudoranges through a least square estimator for the whole considered trajectory, while Figure 3 and Figure 4 show as a function of altitude the GDOP and pseudorange errors, respectively. From Figure 2, we note that the error increases with the altitude with peaks of more than $50 \mathrm{~km}$ and in the last 5 hours and 45 minutes it has a standard deviation of about $6.7 \mathrm{~km}$, which certainly does not satisfy the positioning accuracy requirements for a moon mission which are typically within $1 \mathrm{~km}(3 \sigma)$ (Woodward and Folta, 2009). 
Figure 2 3D normalised positioning error, for GPS C/A, as function of the altitude (see online version for colours)



Figure 3 GDOP as function of the altitude (see online version for colours)

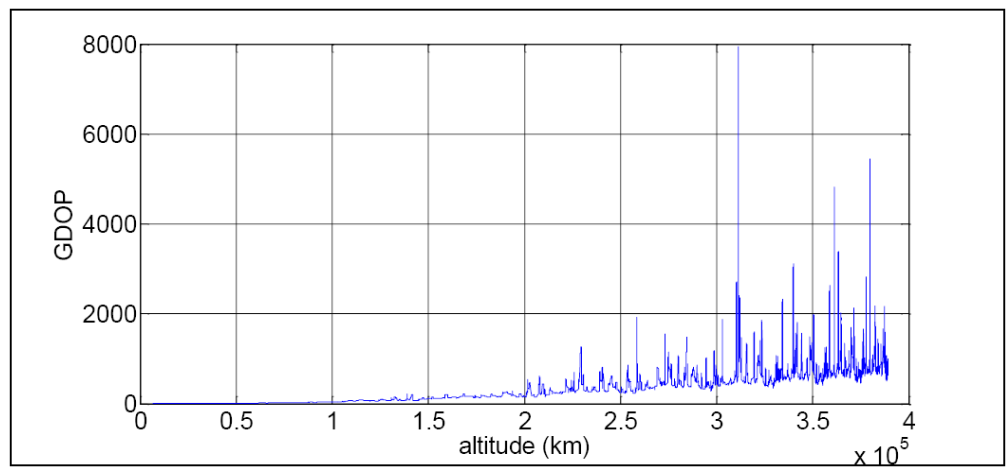

Figure 4 Error on pseudorange as function of the altitude (see online version for colours)

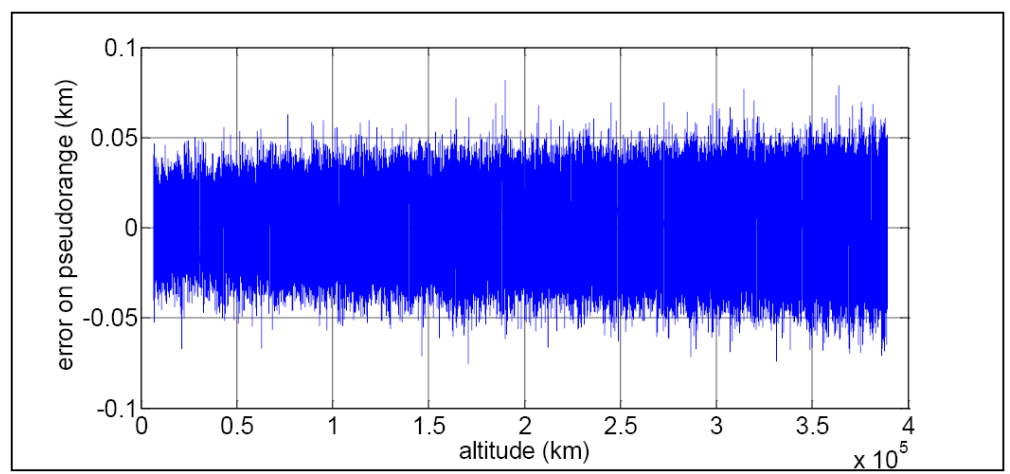

The increasing trend of the error results essentially from two factors: the increasing GDOP value (see Figure 3) due to the more and more limited region of the field of view of the receiver where the GPS satellites are located; and the increasing pseudorange error (see Figure 4) due to the augmentation of the thermal noise code tracking jitter $\sigma_{t D L L}$ which is due to the diminishing received signals power. Such performance curves 
confirm what was reported in other research papers such as Capuano et al. (2014a), Palmerini et al. (2009), and Braasch and de Haag (2006).

\section{Implementation of the orbital filter}

\subsection{Integration in the position domain and in the range domain}

In this study, we investigate and compare the performance of a GPS-based orbital filter for two different input configurations. The first one (denoted 'position-based') uses the standalone GPS least square position and velocity solution as input of the filter; the integration is done in the position-domain and can be considered as 'loose integration' between GPS and orbital forces model. The second one denoted as 'range-based', uses directly pseudorange and pseudorange rate measurements as input of the filter and the un-weighted least square algorithm is replaced by an eight-state Kalman filter that computes user's position and velocity, and receiver's clock offset and drift. In this latter case, we may talk about 'tight integration' between GPS and an orbital forces model, since it is done in the range-domain.

In both configurations due to dissimilar error characteristics, the achievable accuracy is higher than the one obtainable using individually a GPS receiver or an orbital propagator. The position-domain integration includes a standalone GPS receiver that is still independent (fault tolerant system), thus, it is simpler and has a clearer approach since the measurements provided by the receiver are directly position and velocity obtained from pseudorange and pseudorange rate observations by means of a least square estimator. On the other side in such configuration, the GPS input cannot be used if less than four GPS satellites are available but it can provide a higher availability of the total system using the orbital propagator alone as interpolator between GPS observations. The range-domain integration is more complex and requires a less transparent approach but it provides higher availability of the total system because less than four GPS satellites can be used as useful observations to provide a navigation solution. In both configurations, the navigation solution can be used to provide an aiding to the GPS signal processing engine in order to improve its performance and thereby also improve the GPS solution's availability.

\subsection{Extended Kalman filter}

The algorithm, which iteratively repeats itself, consists of two phases: the prediction of the measurements by using a system model that propagates the state and the update through the real measures. If a dynamic system is described by the set of nonlinear differential equations

$$
\dot{x}(t)=f(x, t)
$$

where $x$ is the state vector, the a priori state $x_{k}^{-}$can be obtained by integrating the equations (5.1). This yields to (Groves, 2013):

$$
x_{k}^{-}=x_{k-1}^{+}+\int_{k-1}^{k} f(x, t) d t
$$


with $x_{k-1}^{+}$that denotes the last state estimate. The uncertainties on the propagated state are described by the covariance matrix $P_{k}^{-}$(Groves, 2013):

$$
P_{k}^{-}=\Phi_{k-1}^{k} \cdot P_{k-1}^{+} \cdot\left(\Phi_{k-1}^{k}\right)^{T}+Q_{k}
$$

In (5.3), $Q_{k}$ represents the covariance matrix of the error on the process, $\Phi_{k-1}^{k}$ is the state transition matrix obtained from (Groves, 2013)

$$
\Phi_{k-1}^{k}=e^{A_{k-1}\left(t_{k}-t_{k-1}\right)}
$$

where

$$
A_{k-1}=\left.\frac{\partial}{\partial x} f(x, t)\right|_{x=x_{k-1}^{+}}
$$

is the linearised system matrix about the a posteriori state vector estimate at time $t_{k-1}$.

In the update step, the propagated state vector is used to predict the measurements

$$
z_{k}=h\left(x_{k}^{-}, t_{k}\right)
$$

where $h(x, t)$ are the observation functions. These measures are supposed to be affected by a white Gaussian noise described by the covariance matrix $R_{k}$. The updated state vector can be computed from (Groves, 2013)

$$
\begin{aligned}
& x^{+}\left(t_{k}\right)=x^{-}\left(t_{k}\right)+K_{k} \cdot\left(z_{k}-h\left(x_{k}^{-}, t_{k}\right)\right) \\
& P_{k}^{+}=\left(I-K_{k} H_{k}\right) \cdot P_{k}^{-}
\end{aligned}
$$

$P_{k}^{+}$is the covariance matrix of the error on the estimated state. The term $K_{k}$ in (5.7) and (5.8) denotes the Kalman gain, it is given by the following expression (Groves, 2013):

$$
K_{k}=P_{k}^{-} H_{k}^{T} \cdot\left(H_{k} P_{k}^{-} H_{k}^{T}+R_{k}\right)^{-1}
$$

with $H_{k}$ that is the Jacobian matrix of the observation functions linearised about the propagated state.

\subsection{State vector}

The state vector $\boldsymbol{x} \in\left\{\boldsymbol{x}_{p}, \boldsymbol{x}_{\boldsymbol{r}}\right\}$ contains the set of parameters describing the system. For the position-based orbital filter, the state vector $x_{p}$ contains the position and velocity components of the receiver, i.e.,

$$
\boldsymbol{x}_{p}=\left[\begin{array}{llllll}
x & y & z & u & v & w
\end{array}\right]^{T}
$$

For the range-based orbital filter, the state vector $\boldsymbol{x}_{r}$ is composed by eight elements: the position and velocity components of the receiver as well as the receiver's clock offset $b$ and $\operatorname{drift} \dot{b}$, i.e., 


$$
\boldsymbol{x}_{r}=\left[\begin{array}{llllllll}
x & y & z & b & u & v & w & \dot{b}
\end{array}\right]^{T}
$$

\subsection{Measurement vector}

For the position-based orbital filter, the measurement vector $z \in\left\{z_{p}, z_{r}\right\}$ can be written as:

$$
\boldsymbol{z}_{\boldsymbol{p}}=\left[\begin{array}{l}
\boldsymbol{x}_{G P S} \\
\boldsymbol{v}_{G P S}
\end{array}\right]
$$

where $\boldsymbol{x}_{G P S}$ and $\boldsymbol{v}_{G P S}$ are respectively the position and velocity vectors computed through a least square estimator.

For the range-based orbital filter, the measurement vector is:

$$
\boldsymbol{z}_{\boldsymbol{r}}=\left[\begin{array}{c}
\boldsymbol{\rho}_{G P S} \\
\dot{\boldsymbol{\rho}}_{G P S}
\end{array}\right]
$$

where $\boldsymbol{\rho}_{G P S}$ and $\dot{\boldsymbol{\rho}}_{G P S}$ are respectively the pseudoranges and pseudorange rates of the available GPS satellites.

\subsection{Process model}

In the Kalman filter estimation, measurements are fused with a mathematical model of the dynamics in order to obtain an optimal solution. During a MTO, a spacecraft is influenced by a wide set of perturbations that make its orbit different from the osculating orbit. Depending on the altitude, each perturbation can have a weaker or stronger effect on the spacecraft dynamics. For instance, the main orbital forces that influence the motion of a satellite in LEO are different from those that affect a spacecraft orbiting around the moon. Hence, the orbital filter uses different process models based on the altitude of the receiver, in order to lower the computational burden. Indeed, it would not be meaningful to model, for instance, the gravitational perturbation due to the sun when the spacecraft is in LEO, as well as modelling the spherical harmonics of the Earth gravitational potential when the receiver is close to the moon.

The main acceleration for a spacecraft orbiting around the Earth is defined by (Montenbruck and Gill, 2000):

$$
\ddot{\boldsymbol{r}}=-\frac{\mu_{\oplus} \boldsymbol{r}}{r^{3}}
$$

where $\boldsymbol{r}$ is the position vector of the spacecraft with respect to the ECI frame and $r$ is its module; $\mu_{\oplus}=G M_{\oplus}$ is the Earth's planetary parameter equal to $398,600.4405 \mathrm{~km}^{3} / \mathrm{s}^{2}$.

Several additional accelerations, which can be considered as perturbation of the osculating orbit, have to be included in the model to increase the accuracy of the dynamics estimation. In particular, the process model (that corresponds to an orbital propagator) includes: spherical harmonics of Earth gravitational potential up to 6th degree and 6th order in LEO; spherical harmonics up to 2nd degree and 2nd order, SRP, and the gravitational perturbations due to the sun and the moon from LEO to $50,000 \mathrm{~km}$ altitude; and above 50,000 km, SRP and lunar and solar third body perturbations. All the perturbations have been modelled according to Montenbruck and Gill (2000), except for 
the SRP which has been modelled following the method presented in Battin (1987) [see Basile (2015) for more details].

Note that the atmospheric resistance in LEO is not modelled in order to save computational burden at the expense of an inaccuracy of the mathematical propagation since at this altitude the GPS measurements are very accurate and a precise orbit propagation is not required. In a future enhancement, in order to increase the accuracy, the atmospheric resistance could be modelled as well, if enough computational resources are available.

\subsubsection{Accuracy of the process model}

The accuracy of the implemented propagator used as process model is shown in Figure 5 for the full MTO trajectory. The 3D position error with respect to the reference orbit is up to $280 \mathrm{~km}$.

Figure 5 Implemented propagator drift respect to reference orbit (see online version for colours)



\subsection{Observation functions}

When measurements are provided by the GPS receiver, they are predicted by the process model of the filter as follows:

$$
\begin{aligned}
& \rho^{-}=h_{\rho}\left(\boldsymbol{x}^{-}\right)=\sqrt{\left(x_{\text {sat }}-x\right)^{2}+\left(y_{\text {sat }}-y\right)^{2}+\left(z_{\text {sat }}-z\right)^{2}}+b \\
& \dot{\rho}^{-}=h_{\dot{\rho}}\left(\boldsymbol{x}^{-}\right)=\left(\boldsymbol{v}_{\text {sat }}-\boldsymbol{v}\right) \cdot \boldsymbol{a}+\dot{b}
\end{aligned}
$$

where $x_{\text {sat }}, y_{\text {sat }}, z_{\text {sat }}$ are the position's components of the GPS satellite that is transmitting the signal, $x, y, z$ are the predicted user's position components, $b$ is the receiver's clock offset, $\boldsymbol{v}_{\text {sat }}$ and $\boldsymbol{v}$ are, respectively, the velocity vector of the transmitting GPS satellite and of the spacecraft, $\dot{b}$ is the clock's drift, and $\boldsymbol{a}$ is the LOS vector from the user to the GPS satellite.

Thus, the observation vector $z^{-}$corresponds to:

$$
\boldsymbol{z}^{-}=\boldsymbol{h}\left(\boldsymbol{x}^{-}\right)=\left[\begin{array}{llllllll}
\rho_{1}^{-} & \rho_{2}^{-} & \cdots & \rho_{n}^{-} & \dot{\rho}_{1}^{-} & \dot{\rho}_{2}^{-} & \cdots & \dot{\rho}_{n}^{-}
\end{array}\right]^{T}
$$




\subsection{State transition matrix computation}

The state transition matrix aims to propagate the covariance matrix of the error on the state estimate as shown in equation (5.3) and it is equal to an exponential function of the system matrix linearised about the state vector estimate $A$ [see equation (5.4)]. However, it can be approximated as (Groves, 2013)

$$
\Phi_{k-1}=e^{A_{k-1}\left(\tau_{s}\right)} \cong\left(I+A_{k-1} \tau_{s}\right),
$$

where $I$ is the unit matrix and $\tau_{s}$ is the propagation interval.

In order to compute $A$ [and then to linearise the process $f(x, t)$ about the updated state as shown in equation (5.5)], the complex-step derivative approximation is adopted. This method has been used in many studies such as Anderson et al. (2001), Martins et al. (2000), Lai and Crassidis (2006) and Martins et al. (2003).

\subsection{Observation matrix}

The observation matrix $H$ is defined as the Jacobian of the observation functions (5.15) as follows:

$$
H=\left.\frac{\partial \boldsymbol{h}(\boldsymbol{x})}{\partial \boldsymbol{x}}\right|_{\boldsymbol{x}=\boldsymbol{x}^{-}}
$$

For the position-based orbital filter, the measurements vector includes the same physical quantities that define the state vector: position and velocity, in fact:

$$
\boldsymbol{z}_{p}=H_{p} \boldsymbol{x}_{p}=\left[\begin{array}{llllll}
x & y & z & u & v & w
\end{array}\right]^{T}
$$

Hence, the observation matrix is equal to a 6 order identity matrix:

$$
H_{p}=\left[\begin{array}{llllll}
1 & 0 & 0 & 0 & 0 & 0 \\
0 & 1 & 0 & 0 & 0 & 0 \\
0 & 0 & 1 & 0 & 0 & 0 \\
0 & 0 & 0 & 1 & 0 & 0 \\
0 & 0 & 0 & 0 & 1 & 0 \\
0 & 0 & 0 & 0 & 0 & 1
\end{array}\right]
$$

For the range-based orbital filter, the measurement vector contains pseudoranges and pseudorange rates and the state vector also includes the receiver's clock initial bias and drift.

For $n$ pseudorange and pseudorange rate observations, the observation matrix $H_{r}$ is the following $2 n \times 8$ matrix: 


$$
H_{r}=\left[\begin{array}{cccccccc}
a_{x 1} & a_{y 1} & a_{z 1} & 1 & 0 & 0 & 0 & 0 \\
a_{x 2} & a_{y 2} & a_{z 2} & 1 & 0 & 0 & 0 & 0 \\
\vdots & \vdots & \vdots & \vdots & \vdots & \vdots & \vdots & \vdots \\
a_{x n} & a_{y n} & a_{z n} & 1 & 0 & 0 & 0 & 0 \\
0 & 0 & 0 & 0 & a_{x 1} & a_{y 1} & a_{z 1} & 1 \\
0 & 0 & 0 & 0 & a_{x 2} & a_{y 2} & a_{z 2} & 1 \\
\vdots & \vdots & \vdots & \vdots & \vdots & \vdots & \vdots & \vdots \\
0 & 0 & 0 & 0 & a_{x n} & a_{y n} & a_{z n} & 1
\end{array}\right]
$$

where $a_{x i}, a_{y i}, a_{z i}$ are the component of the user-to-satellite LOS.

\subsection{Adaptive architecture of the system}

A Kalman filter computes an optimal estimate by weighting the process and the measures through their variance-covariance matrices. As seen in Section 4, during a MTO, once the receiver is above the GPS constellation, the GPS accuracy strongly decreases with the altitude: both pseudorange error and GDOP increase as the receiver moves further from the GPS constellation. Therefore, if the variance-covariance matrix of the measurements $R$ is kept constant, the filter cannot be tuned properly during the full trajectory. Figure 6 shows the position-domain filtered solution accuracy when the covariance matrix of the measurements $R$ is set as constant and tuned for the LEO portion of the trajectory, while Figure 7 shows the performance obtained when $R$ is set as constant but tuned for the last part of the trajectory. In the first case, when the tuning is optimised for LEO, the Kalman gain is computed by weighting more the measures (more accurate in LEO) than the process; hence, the estimation error at higher altitude approximately equals the measurements error. In the second case, the filter is tuned as it would operate at higher altitude; and it is clear from Figure 7 that while at high altitude the filter reduces strongly the measurement error, in LEO, the estimation error is even larger.

Figure 6 Orbital filter performance when no adaptivity is used: the $R$ matrix is tuned to work well at low altitude (see online version for colours)

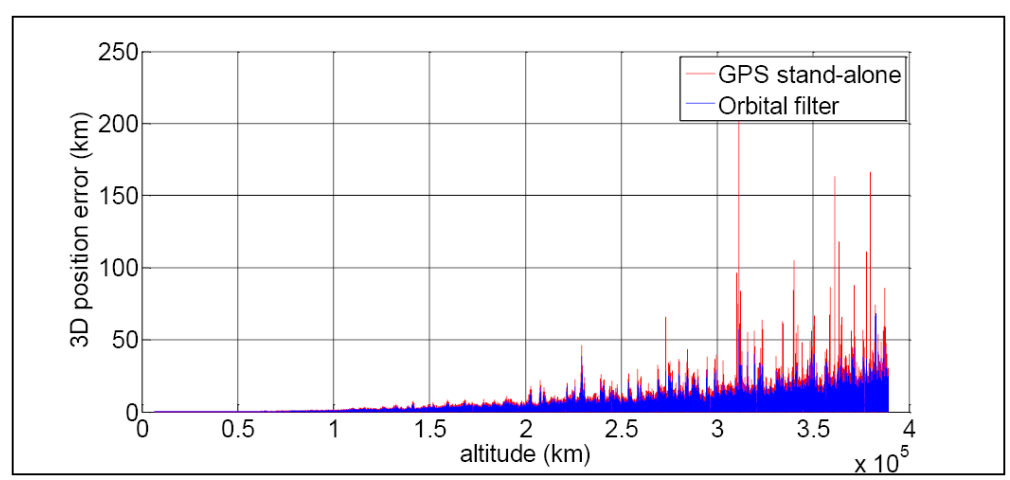


Therefore, the variance-covariance matrix $R$ has to be adapted to the GPS measurements accuracy. Obviously, this strategy is applied in different ways to the different configurations of the orbital filter. In case the measurements vector is composed by position and velocity (integration in the position-domain), the $R$ matrix is a function of the GDOP multiplied by the estimated pseudorange error; instead, in case of pseudorange and pseudorange rates as input of the filter (integration in the range-domain), the $R$ matrix does not include the contribution of GDOP.

Figure 7 Orbital filter performance when no adaptivity is used (zoom on the first 40,000 s): the $R$ matrix is tuned to work properly at high altitude (see online version for colours)

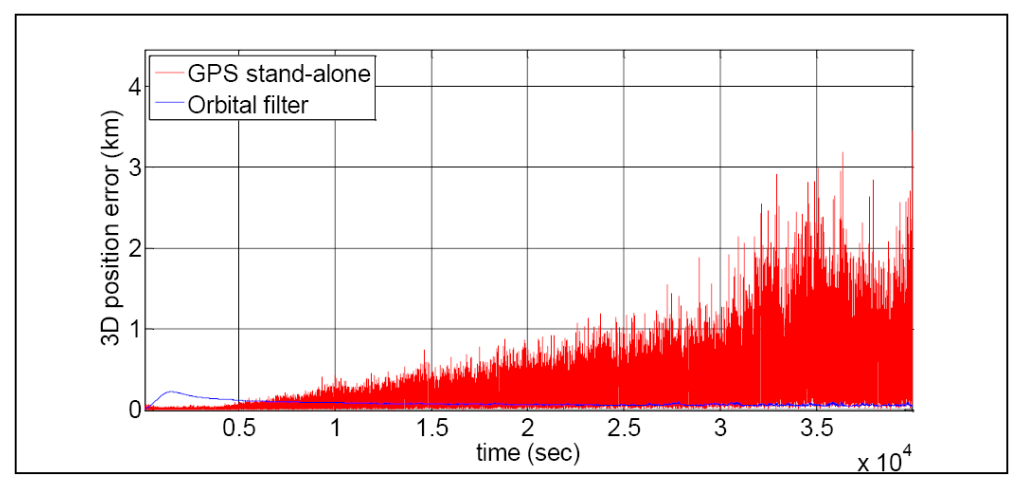

Figure 8 Adaptive strategy (see online version for colours)

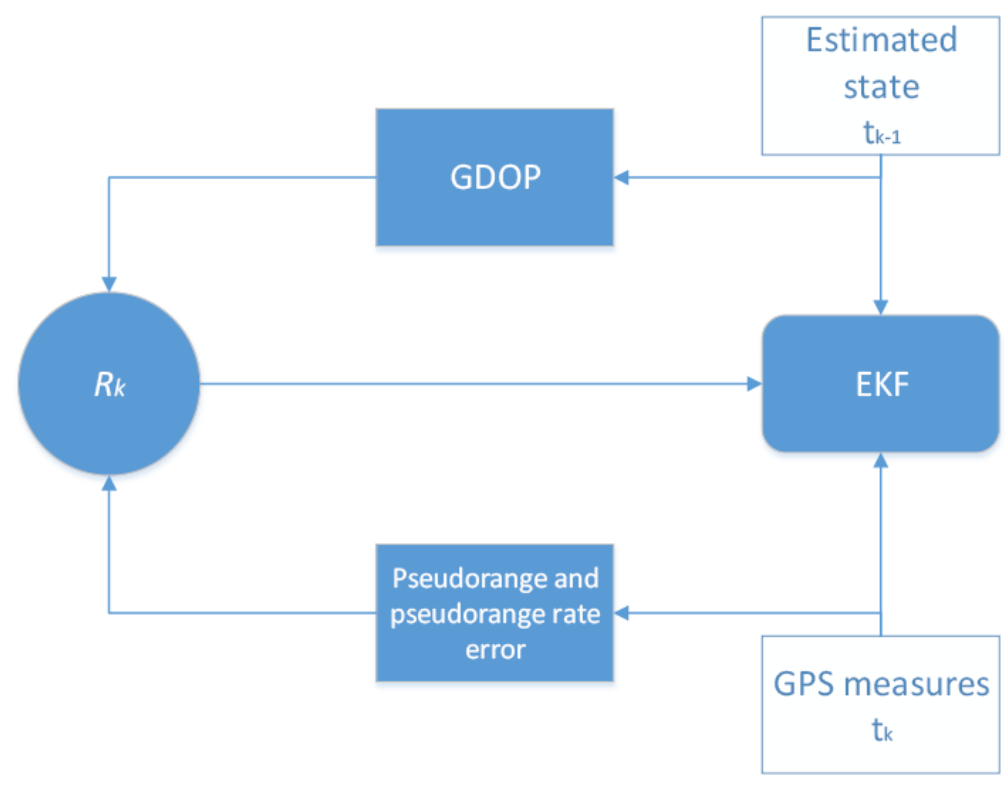

Note: In case the pseudorange is directly processed in the filter, the GDOP does not appears in the computation of the $R$ matrix. 
The adaptive strategy is illustrated in Figure 8. From the estimated state computed at the previous time-step, the filter estimates the GDOP and the noise on both pseudorange and pseudorange rate measures.

In addition, as illustrated in Figure 2, the GPS error may reach very high peaks of hundreds km, due to the corresponding peaks of the GDOP (see Figure 3). For this reason, the orbital filter makes a check of the GDOP computed by means of the estimated state. In case it exceeds a threshold (a value of 1,500 has been set after a tuning), the filter replaces the estimated state with the propagated one. This corresponds to rely completely on the orbital propagator when the relative receiver-GPS satellites geometry, and therefore the observations, is too poor.

\subsection{Simulation test bench}

For both filters, the simulation consists of six steps:

1 Filter initialisation. According to Kalman filter theory, both $P_{0}^{-}$and $x_{0}^{-}$are initialised from known data. This data is obtained from the first fix of the GPS receiver. In order to pass from pseudoranges to position, an un-weighted least square algorithm is used.

2 Measures. Once the initialisation is completed, the filtering loop can start. At each instant of time, pseudoranges and pseudorange rates are computed by using equations (3.1) and (3.2), respectively, from the true ranges and ranges rate. The visible and available satellites are selected based on the signal power level at the receiver position provided by the PosApp software. Each signal is considered available if its power level at the receiver is higher than the threshold of $-169 \mathrm{dBm}$ (assuming $-159 \mathrm{dBm}$ receiver sensitivity and $10 \mathrm{dBi}$ receiver antenna gain).

3 Filter estimation of pseudorange error and GDOP. Prediction of pseudorange and pseudorange rate errors and GDOP are computed by using the observation functions (5.15).

$4 \quad R$ matrix. Pseudorange and pseudorange rate errors and GDOP estimates are used to update the variance-covariance matrix $R$. It is a six order square matrix for the position-based orbital filter, where the mean value of all the computed tracking thermal noises together with the GDOP are included in $R$. For the range-based filter, $R$ is a $2 N$ order square matrix, where $N$ is the number of available satellites. In this case, the GDOP does not have any impact on the measurements, contrary to the position-based filter architecture where the GDOP is directly used to compute the variance-covariance matrix of the measurements error.

$5 E K F$. At this point, the EKF has all it needs to provide the state estimate. Its output is used as input for the following estimation.

6 Computation of errors. Once the filtering loop is over and the trajectory is estimated, the errors can be computed by comparing the estimation to the reference trajectory. 


\section{Results}

\subsection{Orbital filter performance}

Figure 9 and Figure 10 illustrate the 3D position error and the 3D velocity error, respectively for the position-based orbital filter and the range-based one. As expected and discussed above using pseudorange and pseudorange rate as direct input is more efficient. In the last 5 hours and 45 minutes of orbit, the standard deviation of the 3D position error is approximately equal to $430 \mathrm{~m}(1 \sigma)$ for the position-based configuration and to $100 \mathrm{~m}(1 \sigma)$ for the range-based configuration.

Figure 9 GPS L1 C/A-based orbital filter 3D position error for the defined MTO (see online version for colours)

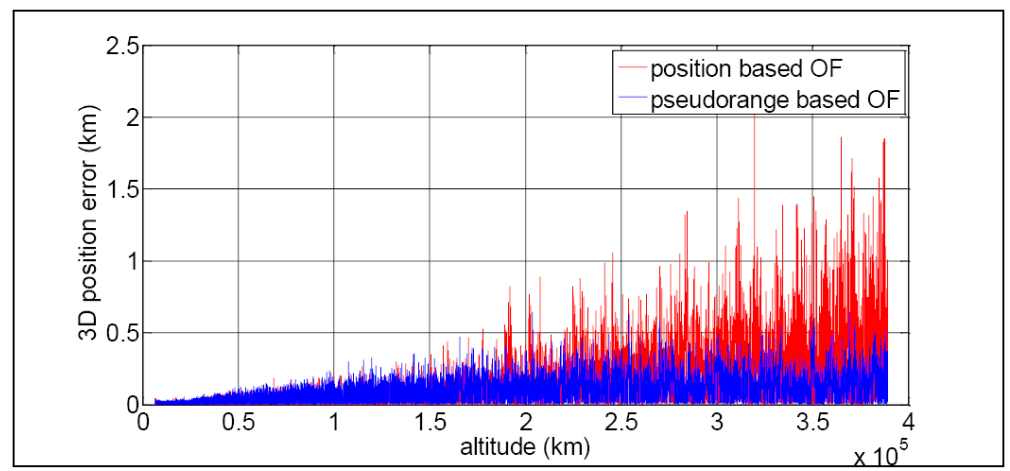

Figure 10 GPS L1 C/A-based orbital filter 3D velocity error for the defined MTO (see online version for colours)

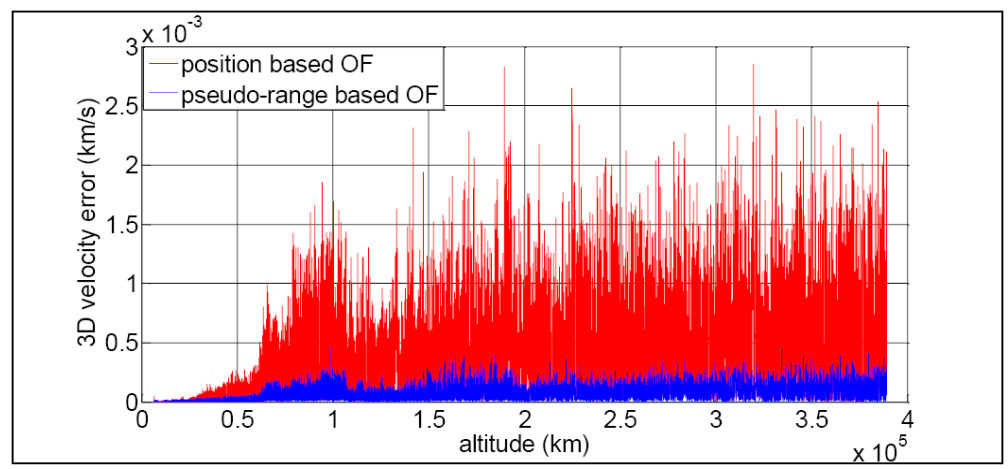

Figure 11 shows the orbital filter performance compared to the standalone GPS performance: using the same scale the error of the orbital filter is not even visible and a zoom is required as shown in Figure 12. 
Figure 11 Comparison between stand-alone GSP measures, position-based orbital filter and pseudo-rage-based orbital filter (see online version for colours)

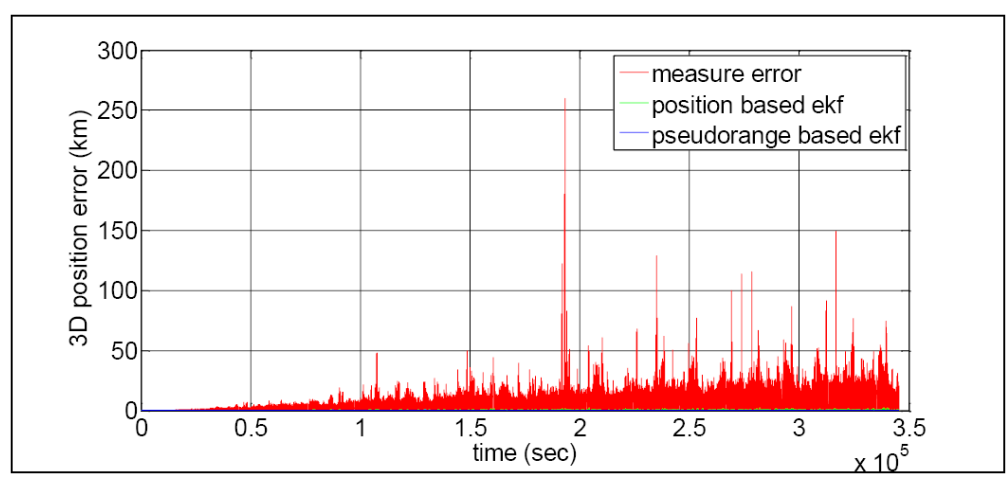

Figure 12 Zoom of comparison between stand-alone GSP measures, position-based orbital filter and pseudo-range-based orbital filter (see online version for colours)

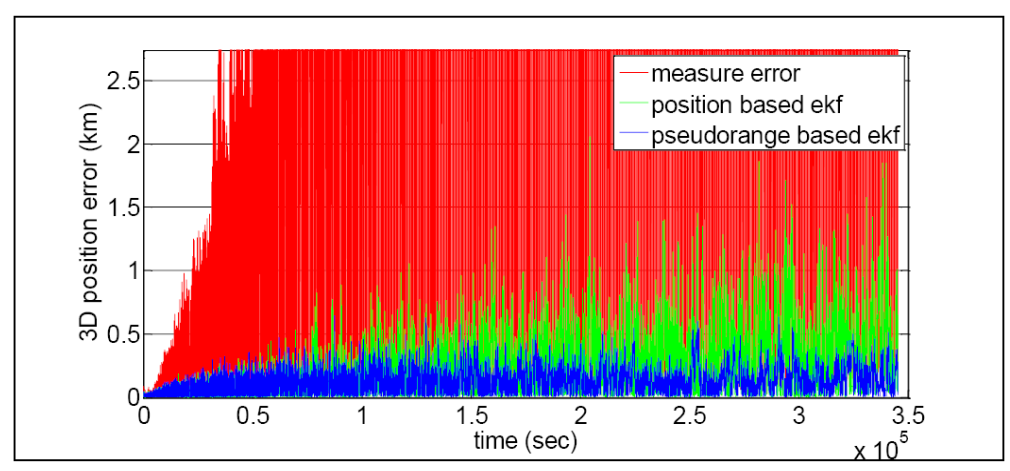

Figure 13 Orbital filter 3D position error when 4 or less than 4 pseudoranges are available (see online version for colours)

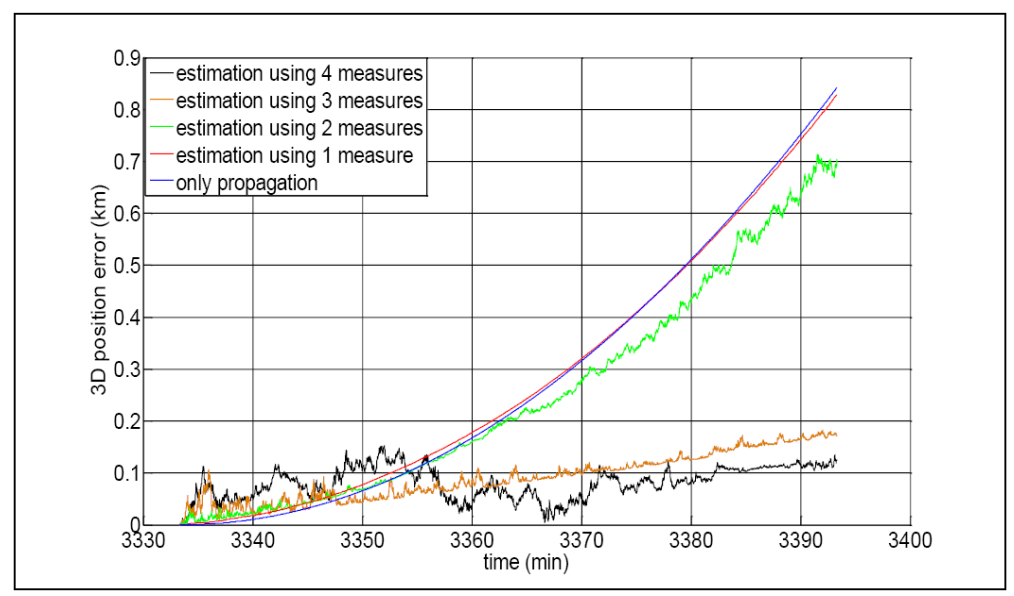


Figure 14 Carrier Doppler orbital filter estimation error for PRN 4 signal for the last 90 minutes of the MTO (see online version for colours)

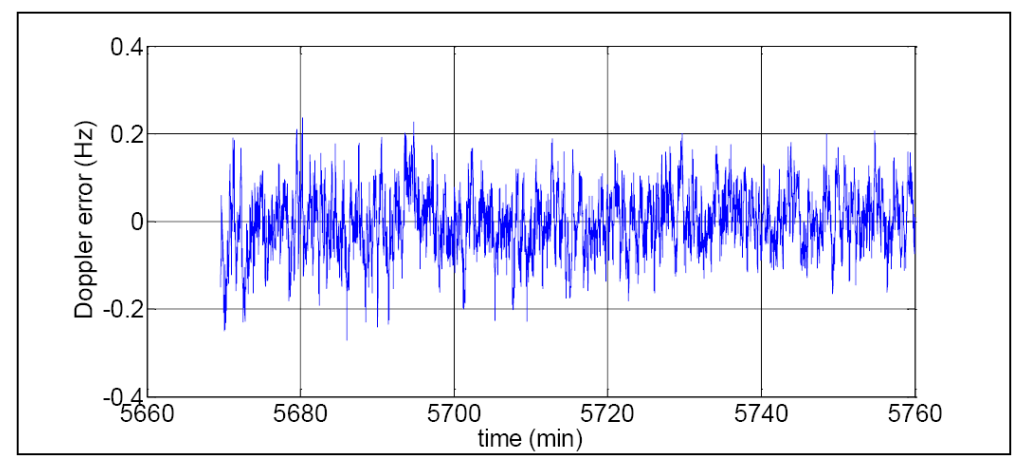

Figure 15 Carrier Doppler rate orbital filter estimation error for PRN 4 signal for the last 90 minutes of the MTO (see online version for colours)



Figure 13 illustrates the efficiency of the range-based orbital filter when a limited number of observations are available. The test has been done for a small portion after the first 3,330 min of the defined MTO and it shows that after a short time interval (about $25 \mathrm{~min}$ ) the orbital filter is already able to provide a 3D position with an error that is smaller than the one obtainable by processing four pseudoranges. In the first $10 \mathrm{~min}$, the only orbital propagation is even more accurate than the fusion with the measurements. This demonstrates the robustness of the orbital propagation filter against poor visibility periods or total signal outages.

Note that the orbital filter can be used not only to improve the navigation performance, but also to provide assistance data for the acquisition and tracking process. The estimation of Doppler and Doppler rate allows to predict the incoming signal's frequency and its variation, thus allowing longer dwell times (for the correlation of the incoming signal with the generated replica in the GPS receiver) for a given signal dynamic or stronger robustness against higher signal dynamics for a given dwell time. If the Doppler and Doppler rate estimation are enough accurate, a much higher sensitivity can be obtained, also in very high dynamics environment such as in LEO when the receiver can reach a relative velocity of several $\mathrm{km} / \mathrm{s}$ with respect to the GPS satellites. The prediction of the GPS visible satellites reduces the number of PRNs that have to be searched in acquisition, reducing the acquisition time. A more detailed study that 
quantifies the required assistance is presented in Capuano et al. (2014a). Figure 14 and Figure 15 respectively show an example of the carrier Doppler and carrier Doppler rate estimation errors when they are computed by using the position and velocity estimated by the range-based orbital filter. Such values can greatly aid the signal processing module both in acquisition and tracking processes as demonstrated in our previous work (Capuano et al., 2014a).

\section{Conclusions}

New GNSS receiver's technologies have allowed to extend the use of GNSS to very challenging applications, such as navigation system for lunar missions, where the GNSS signals are characterised by very low power level at the receiver and the system geometry is poor. This appears to be appealing since using GNSS could yield to significant reduction of the operating cost for such missions.

This paper demonstrates that a relatively accurate and autonomous orbital determination may be performed by means of a GPS-based orbital filter, which provides the navigation solution by filtering GPS observations through a space dynamic model. Here, a comparison between two different configurations of the orbital filter, range-based and position-based, is performed. The most remarkable results are obtained with the range-based architecture: we achieved an accuracy of about $100 \mathrm{~m}$ ( $1 \sigma$ standard deviation) in position at moon altitude.

In future works, the functioning of the range-based orbital filter implementation will be further assessed using our full-constellation GNSS simulator and processing the GPS observations directly with the hardware receiver currently under development in our laboratory, specifically designed for space missions, in order to validate the results obtained so far. The use of GNSS signals from GPS-Galileo combined constellation and use of two frequencies to mitigate possible ionosphere delay will be investigated as well in order to improve the performance. In this context, finally, we will also optimise the error budget on the measurements to have more realistic simulations.

\section{References}

Anderson, K.W., Newman, J.C. and Nielsen, E.J. (2001) 'Sensitivity analysis for Navier-Stokes equations on unstructured meshes using complex variables', AIAA Journal, Vol. 39, No. 1, pp.56-63.

Balbach, O. et al. (1998) Tracking GPS above GPS Satellite Altitude: First Results of the GPS Experiment on the HEO Mission Equator-S, IEEE, Palm Springs, California, USA.

Basile, F. (2015) Implementation of the Orbital Filter in a GNSS Receiver for Lunar Missions, University of Rome La Sapienza, École polytechnique fédérale de Lausanne (EPFL), Rome, Italy.

Battin, R.H. (1987) An Introduction to Mathematics and Methods of Astrodynamics, AIAA Education Series, New York, NY, USA.

Bauer, F. et al. (2006) The GPS Space Service Volume, ION Publications, Fort Worth, TX, USA.

Betz, J.W. and Kolodziejski, K.R. (2000) 'Extended theory of early-late code tracking for a bandlimited GPS receiver', Navigation, Vol. 47, No. 3, pp.211-226.

Borio, D., Sokolova, N. and Lachapelle, G. (2010) 'Doppler measurement accuracy in standard and high-sensitivity GNSS receivers', IET Radar, Sonar \& Navigation, Vol. 5, No. 6, pp.657-665. 
Braasch, M.S. and de Haag, M.U. (2006) 'GNSS for LEO, GEO, HEO and beyond', American Astronautical Society Guidance and Control Conference, Breckenridge, CO, USA, pp.165-194 .

Capuano, V. et al. (2014a) GNSS to Reach the Moon, International Astronautical Federation, Toronto, Canada.

Capuano, V. et al. (2014b) GNSS/INS/Star Tracker Integrated Navigation System for Earth-Moon Transfer Orbit, The Institute of Navigation, Tampa, Florida, USA.

Chiaradia, A.P.M. et al. (2000) Algorithms for On-Board Orbit Determination using GPS $O B O D E-G P S$, DLR-GSOC TN 00-04, Oberpfaffenhofen.

Choi, E.J. et al. (2010) 'Onboard orbit determination using GPS observations based on the unscented Kalman filter', Advances in Space Research, Vol. 46, No. 11, pp.1440-1450.

Czopek, F.M. and Shollenberger, S. (1993) Description and Performance of the GPS Block I and II L-Band Antenna and Link Budget, The Institute of Navigation, Salt Lake City, UT.

Global Positioning System Directorate (2013) Interface Specification IS-GPS-200, USA.

Groves, P.D. (2013) Principles of GNSS, Inertial, and Multisensor Integrated Navigation Systems, Artech House, Boston, MA, USA.

Habib, T.M.A. (2013) 'Simultaneous spacecraft orbit estimation and control based on GPS measurements via extended Kalman filter', The Egyptian Journal of Remote Sensing and Space Sciences, Vol. 16, No. 1, pp.11-16.

Kaplan, E.D. and Hegarty, C.J. (2006) Understanding GPS: Principles and Applications, Artech House, Boston, MA, USA.

Klobuchar, J. (1987) 'Ionospheric time-delay algorithm for single-frequency GPS users', Aerospace and Electronic Systems, IEEE Transactions on, AES, Vol. 23, No. 3, pp.325-331.

Lai, K.L. and Crassidis, J.L. (2006) Generalizations of the Complex-Step Derivative Approximation, American Institute of Aeronautics and Astronautics, Reston, VA, USA.

Litva, J. (1996) Digital Beamforming in Wireless Communications, Artech House, Boston, MA, USA.

Manzano-Jurado, M. et al. (2014) Use of Weak GNSS Signals in a Mission to the Moon, IEEE, Nordwijk, Netherlands.

Martins, J.R.R.A., Kroo, I.M. and Alonso, J.J. (2000) An Automated Method For Sensitivity Analysis Using Complex Variables, AIAA, Reno, Nevada, USA.

Martins, J.R.R.A., Sturdza, P. and Alonso, J.J. (2003) 'The complex-step derivative approximation', ACM Transactions on Mathematical Software, Vol. 29, No. 3, pp.245-262.

Montenbruck, O. and Gill, E. (2000) Satellite Orbits: Models, Methods, Applications, Springer, Berlin, Germany.

Palmerini, G.B., Sabatini, M. and Perrotta, G. (2009) 'En route to the moon using GNSS signals', Acta Astronautica, Vol. 64, No. 4, pp.467-483.

Powell, T.D. et al. (1999) 'GPS Signals in a Geosynchronous Transfer Orbit: 'Falcon Gold' Data Processing, ION Publications, San Diego, California, USA.

Silva, P.F. et al. (2013) Weak GNSS Signal Navigation to the Moon, ION Publications, Nashville, Tennessee, USA.

Spirent (2012) SimGen Software User Manual, Spirent Communications, Eatontown, NJ, USA.

Van Diggelen, F. (2009) A-GPS: Assisted GPS, GNSS and SBAS, Artech House, Boston, MA, USA.

Woodward, M. and Folta, D.a.W.D. (2009) 'ARTEMIS: the first mission to the lunar libration orbits', 21st International Symposium on Space Flight Dynamics, Toulouse, France.

Zhou, N. (2004) Onboard Orbit Determination Using GPS Measurements for Low Orbit Satellites, Qeensland University of Technology, Brisbane, Australia. 